\title{
First American Stand of Paramecium novaurelia and Intra-specific Differentiation of the Species*
}

\author{
Ewa PRZYBoŚ, Sebastian TARCZ and Irina SKOBLO
}

Accepted September 20, 2006

\begin{abstract}
PrZyboś E., TARCZ S., SKoblo I. 2007. First American stand of Paramecium novaurelia and intra-specific differentiation of the species. Folia biol. (Kraków) 55: 53-63.

A stand of Paramecium novaurelia was found in Boston Massachusetts, USA, the first on this continent. Molecular studies (RAPD and sequencing of rRNA [ 3'SSUrRNA-ITS1, 5' LSU rRNA] and COI mtDNA fragments) of $P$. novaurelia strains show intra-specific polymorphism within the species as strain clusters characterized by variable relationships.

Key words: Paramecium aurelia species complex, intra-specific differentiation, breeding system, geographical distribution, RAPD-PCR fingerprinting, sequencing of SSU-ITS1, LSU rRNA and COI mtDNA fragments.

Ewa PRZYBoŚ, Sebastian TARCZ, Department of Experimental Zoology, Institute of Systematics and Evolution of Animals, Polish Academy of Sciences, Stawkowska 17, 31-016 Kraków, Poland.

E-mail:przybos@isez.pan.krakow.pl

E-mail:starcz@isez.pan.krakow.pl

Irina SKOBLO, Biological Research Institute, St. Petersburg State University, Old Peterhof, Oranienbaumskoye sch. 2, 198904 St. Petersburg, Russia.

E-mail: aurelia@fromru.com
\end{abstract}

Among the 15 species of the Paramecium aurelia complex known world-wide (SONNEBORN 1975; AUFDERHEIDE et al. 1983), the majority also occur in the USA. The occurrence of some $(P$. undecaurelia and $P$. sonneborni $)$ seems to be limited to this territory, other species ( $P$. primaurelia, $P$. biaurelia, $P$. tetraurelia, $P$. sexaurelia) are cosmopolitan (SONNEBORN 1975; PRZYBOŚ \& FOKIN 2000) or confined also to other territories (e.g. P. septaurelia, cf PRZYBOŚ et al. 2004, 2005).

P. novaurelia was regarded as restricted to Europe alone (SONNEBORN 1975) and a dominant species there (PRZYBOŚ \& FOKIN 2000), later (PRZYBOŚ 1998) it was also recorded in Asia (Turkey, Anatolian Upland). This species has never been found again outside of Europe, in spite of numerous studied samples originating from different places around the world (cf PRZYBOŚ et al. 2006a). This is the first American record of $P$. novaurelia, identified in a sample collected in Boston, USA. A comparative analysis of molecular features of the American, European, and Asiatic strains of this species is also performed.

\section{Material and Methods}

\section{Material}

The strains designated USB1-4 were collected by I. Skoblo in Boston, USA from a natural ponds in 1994; the YR strain originated from Yellowstone National Park, Yellowstone River, USA, the water sample was collected by C. Vanini in 2004, and the strain was established by S. Fokin; the BR strains from Baton Rouge, USA were collected by S. Fokin in 2004.

\section{Methods}

\section{Culturing and identification of paramecia}

Species of the $P$. aurelia complex were cultured and identified according to the methods of SONNEBORN (1970). Paramecia were cultivated on a lettuce medium inoculated with Enterobacter aerogenes. Clones mature for conjugation were mated with the reactive mating types of standard strains of known species. The following standard strains 
were used: strain 90 of $P$. primaurelia; strain Rieff, Scotland of P. biurelia; strain 87 of $P$. pentaurelia; strain 510 of $P$. novaurelia.

\section{Strain crosses}

In the intra and inter-strain crosses, the $F_{1}$ generation was obtained by conjugation and $F_{2}$ by autogamy (using the method of daily isolation lines). The occurrence of the desired stage of autogamy (specimens at the stage of two macronuclear anlagen) was examined on preparations stained with aceto-carmine. Survival of clones in both generations was estimated as percentages. According to CHEN (1956), clones can be considered as surviving after passing 6-7 fissions during 72 hours after separation of partners of conjuga- tion or postautogamous caryonids. The methods were described in detail in PRZYBOŚ (1975).

3. Methods used in molecular studies

Paramecium genomic DNA was isolated $(200 \mu 1$ of cell culture was used for DNA extraction) from vegetative cells at the end of the exponential phase using the Qiamp DNA Kit (Qiagen ${ }^{\mathrm{TM}}$, Germany) as described by PRZYBOŚ et al. (2003a). All strains used for analysis are listed in Table 1.

\section{a. Amplification of ribosomal DNA (rDNA)}

The primers used for PCR reactions are listed in Table 2, they amplified two regions of rDNA: the 3' end of SSU rRNA - the ITS1 fragment (about $300 \mathrm{bp}$ ) and the 5' end of a fragment of LSU (450bp). One of the primers - LSU_R has the same sequence as in JEROME and LYNN (1996). The

Table 1

Paramecium spp. strains used in molecular studies

\begin{tabular}{|c|c|c|c|c|c|c|}
\hline \multirow{2}{*}{ Species } & \multirow{2}{*}{$\begin{array}{c}\text { Strain } \\
\text { designation }\end{array}$} & \multirow{2}{*}{ Geographical origin } & \multirow{2}{*}{ References } & \multicolumn{3}{|c|}{ Accesion numbers } \\
\hline & & & & ITS1 & 5'LSU & $\mathrm{COI}$ \\
\hline P. novaurelia & 510 & $\begin{array}{l}\text { Great Britain, } \\
\text { Edinburgh }\end{array}$ & BEALE \& SCHNELLER 1954 & DQ837976 & DQ837974 & DQ837975 \\
\hline P. novaurelia & $\mathrm{CVH}$ & $\begin{array}{l}\text { Czech Republic, } \\
\text { Eastern Sudetes Mts, } \\
\text { Nizky Jesenik Mts }\end{array}$ & PRZYBOŚ \& KOMALA 1992 & DQ837980 & DQ837978 & DQ837979 \\
\hline P. novaurelia & FLU & $\begin{array}{l}\text { France, } \\
\text { Lafilorierre }\end{array}$ & $\begin{array}{l}\text { BEALE \& SCHNELLER } \\
1954\end{array}$ & DQ837983 & DQ837981 & DQ837982 \\
\hline P. novaurelia & PB & $\begin{array}{l}\text { Poland, } \\
\text { Białowieża }\end{array}$ & KOMALA et al. 1960 & DQ837986 & DQ837984 & DQ837985 \\
\hline P. novaurelia & PO & $\begin{array}{l}\text { Poland, } \\
\text { Carpathians, Orava Valley, } \\
\text { Lipnica Wielka }\end{array}$ & PRZYBOŚ \& KOMALA 1996 & DQ837989 & DQ837987 & DQ837988 \\
\hline P. novaurelia & PSO & $\begin{array}{l}\text { Poland, } \\
\text { Sudetes Mts, } \\
\text { Orlickie Plateau, Kudowa }\end{array}$ & KOMALA \& PRZYBOŚ 1989 & DQ837992 & DQ837990 & DQ837991 \\
\hline P. novaurelia & SB & $\begin{array}{l}\text { Spain, } \\
\text { Pyrenees, } \\
\text { las Bassotes village }\end{array}$ & PRZYBOŚ 1991 & DQ837995 & DQ837993 & DQ837994 \\
\hline P. novaurelia & TB & $\begin{array}{l}\text { Turkey, } \\
\text { Beysahir }\end{array}$ & PRZYBOŚ 1998 & DQ837998 & DQ837996 & DQ837997 \\
\hline P. novaurelia & USB & $\begin{array}{l}\text { United States, } \\
\text { Boston }\end{array}$ & Present paper & DQ838001 & DQ837999 & DQ838000 \\
\hline P. caudatum & $\mathrm{PC}$ & $\begin{array}{l}\text { Cyprus, } \\
\text { Akamas } \\
\end{array}$ & TARCZ et al. 2006 & DQ207387 & DQ207375 & DQ837977 \\
\hline
\end{tabular}

Table 2

Primers used in this study

\begin{tabular}{|l|c|c|l||}
\hline \multirow{2}{*}{ Amplified region } & Primer & Sequence 5'-3' & \multicolumn{1}{|c||}{ References } \\
\hline \hline \multirow{2}{*}{ 3'SSU rDNA - ITS1 } & ITS1_F & 5'-TAAACCTTATCACTTAGAGGA-3 & TARCZ et al. 2006 \\
\cline { 2 - 5 } & ITS1_R & 5'-CGAAAATCTAATGTCTCGCA-3' & TARCZ et al. 2006 \\
\hline \multirow{2}{*}{ 5'LSU rDNA } & LSU_F & 5'-CCCGTATTTGGTTAGGACT-3' & TARCZ et al. 2006 \\
\cline { 2 - 5 } & LSU_R & 5'-TTGGTCCGTGTTTCAAGACG-3' & JEROME \& LYNN, 1996 \\
\hline \multirow{2}{*}{ CO I } & CoxL11058 & 5'-TGATTAGACTAGAGATGGC-3' & BARTH et al. 2006 \\
\cline { 2 - 5 } & CoxH10176 & 5'-GAAGTTTGTCAGTGTCTATCC-3' & BARTH et al. 2006 \\
\hline
\end{tabular}


other primers were constructed using Oligoanalyzer 3.0 (http://scitools.idtdna.com/analyzer/). Primer construction was based on data from GenBank (Accesion numbers: X03772 - for ITS1_F primer, and $A Y 833401$ - for ITS1 R). Primer LSU F was constructed based on unpublished sequences of the ITS2 - 5'LSU fragment of Paramecium dodecaurelia. PCR amplification was carried out in a final volume of $30 \mu 1$ containing: $2 \mu 1$ of DNA, $1.5 \mathrm{U}$ Taq-Polymerase (Qiagen ${ }^{\mathrm{TM}}$, Germany), $0.6 \mu 110 \mathrm{mM}$ of each primer, 10x PCR buffer, $0.6 \mu 1$ of $10 \mathrm{mM}$ dNTPs in a T-personal thermocycler (Biometra $\mathrm{GmbH}$, Germany). The amplification protocol consisted of initial denaturation at $94^{\circ} \mathrm{C}$, followed by $34 \mathrm{cy}-$ cles of denaturation at $94^{\circ} \mathrm{C}$ for $45 \mathrm{~s}$, annealing at $50^{\circ} \mathrm{C}$ for $60 \mathrm{~s}$, and extension at $72^{\circ} \mathrm{C}$ for $60 \mathrm{~s}$, with final extension at $72^{\circ}$ for $5 \mathrm{~min}$. After amplification the PCR products were electrophoresed in $1 \%$ agarose gels for $45 \mathrm{~min}$ at $85 \mathrm{~V}$ with a DNA molecular weight marker (VI TM Roche, France).

b. Amplification of a fragment of mitochodrial cytochrome oxidase (COI)

To amplify the CO I region (880bp) of mitochonrial DNA, Cox L and Cox $\mathrm{H}$ primers were used (according to BARTH et al. 2006). PCR amplification was carried in the same volume as in the case of rDNA regions (see above), and the protocol followed BARTH et al. (2006). After amplification, the PCR products were electrophoresed in $1 \%$ agarose gels for $45 \mathrm{~min}$ at $85 \mathrm{~V}$ with a DNA molecular weight marker (VI TM Roche, France).

\section{c. Sequencing}

$30 \mu 1$ of each PCR product was separated on a $1.8 \%$ agarose gel $(100 \mathrm{~V} / 60 \mathrm{~min})$. Then, the band representing the examined fragment was cut out and transferred into an $1.5 \mathrm{ml}$ Eppendorf tube. $\mathrm{Pu}$ rification was performed according to the Qiaquick Gel Extraction Kit TM protocol (Qiagen). Cycle sequencing was done in both directions using the BigDye Terminator v3.1 $1^{\mathrm{TM}}$ chemistry (Applied Biosystems, USA). Sequencing products were precipitated using sodium acetate/ethanol and separated on an ABI PRISM 377 DNA Sequencer $^{\mathrm{TM}}$ (Applied Biosystems, USA).

\section{d. Data analysis}

Sequences were examined using Chromas Pro (Technelysium ${ }^{\mathrm{TM}}$, Australia). Alignment and consensus of the study sequences was performed using ClustalW (THOMPHSON et al. 1994) in the BioEdit program (HALL 1999). Phylogenetic trees were constructed for the studied fragments in Mega version 3.1 (KUMAR et al. 2004), using NJ (Neighbor-joining method) (SAITOU \& NEI 1987) and MP (Maximum Parsimony). The NJ analysis was performed using a KIMURA 2-parameter correction model (KIMURA 1980) by bootstrapping with 100 replicates (FELSENSTEIN 1985). The MP analysis was evaluated with Min-mini heuristic parameter (level $=2$ ) and bootstrapping with 100 replicates.

RAPD-PCR fingerprint method was generally performed in accordance with STOECK \& SCHMIDT (1998), details are described in PRZYBOŚ et al. (2003a). RAPD-PCR was performed with a 10mer random primer Ro-460 04 (Roth, Karsruhe, Germany), with nucleotide sequence: 5' -GCAGAGAAGG- 3', using Taq polymerase (Qiagen). This primer was selected from STOECK \& SCHMIDT (1998) after testing several dozen oligonucleotide primers because it gave "robust band patterns" in the $P$. aurelia species complex. It was also used in other studies carried out on the $P$. aurelia species complex (STOECK et al. 1998, 2000) and on P. jenningsi strains (PRZYBOŚ et al. 1999, 2003a; SKOTARCZAK et al. 2004 a, b) and P. schewiakoffi (FOKIN et al. 2004). RAPD-PCR was done in a Biometra thermocycler, products of PCR reactions were separated by electrophoresis in $1.5 \%$ agarose gels for $3.5 \mathrm{~h}$ at $85 \mathrm{~V}$ together with a molecular weight marker $\mathrm{VI}^{\mathrm{TM}}$ (Roche ${ }^{\mathrm{TM}}$, France), then stained with ethidium bromide and visualized in UV light. The images were stored in computer memory using the Scion Image $^{\mathrm{TM}}$ program (Scion Corporation ${ }^{\mathrm{TM}}$, USA). Three repetitions of the PCR reaction were performed in order to assess the reproducibility of the data. Analysis of similarity was carried out by comparing the molecular mass of DNA band patterns obtained by the RAPD method (the Bio1D++ ${ }^{\mathrm{T} M}$ program, Vilbert Lourmat, France) according to the NEI and LI (1979) similarity coefficient, dendrograms were produced using the UPGMA (unweighted pair group match average) algorithm.

\section{Results}

\section{Strain identification based on mating reaction}

The strain from Boston, USA (USB1) originating from population $\mathrm{AB} 8$ was identified as $P$. novaurelia on the basis of strong conjugation with the standard strain of this species. A high percentage of surviving clones was observed in $\mathrm{F}_{1}(86 \%)$ and $\mathrm{F}_{2}(84 \%)$ generations of inter-strain crosses of the USB1 strain with the standard strain (510) from Scotland. This is the first stand of the species in the USA.

The other strains collected from the same locality and originating from one population $\mathrm{AB} 7$, designated USB2, USB3, and USB4 were identified as $P$. biaurelia on the basis of strong conjugation with the standard strain of the species. 

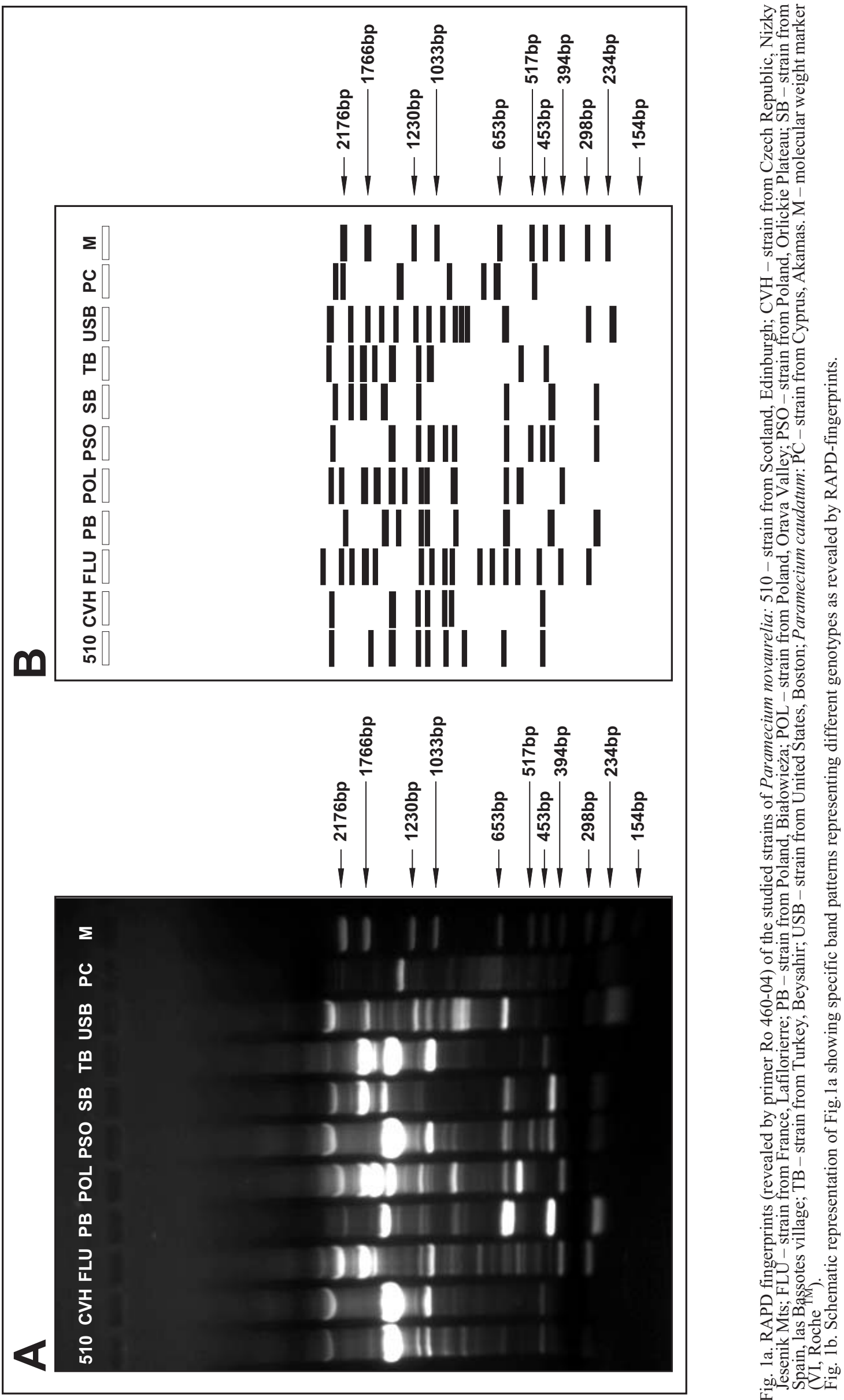
The strain YR, USA was identified as $P$. biaurelia on the basis of strong conjugation with the standard strain of the species. This is a cosmopolitan species.

The paramecia collected in Baton Rouge, Louisiana, USA were identified as Paramecium multimicronucleatum on the basis of analysis of the type and number of their micronuclei (VIVIER 1974) on slides stained using aceto-carmine and Giemsa's stain (after fixation and hydrolysis, cf PRZYBOŚ 1978).

\section{RAPD-PCR analysis}

Fingerprints (band patterns) of the studied $P$. novaurelia strains (the new one from the USA and the other selected strains) and the P. caudatum strain, revealed by DNA amplification with primer Ro 460-04, are presented in Figs 1a,b. Polymorphism within $P$. novaurelia is shown as several groups of genotypes. Strains from Scotland and the Czech Republic (510 and CVH) show about $60 \%$ similarity of band patterns, strains from Poland (PB, POL, PSO) and Spain (SB) compose the next group, the strain from the USA (USB) has a somewhat isolated position as its band pattern shows about $30 \%$ similarity to the previous strain groups, and strains from France and Turkey (FLU and TB) compose the next strain group. The $P$. caudatum band pattern is completely different from the patterns seen in $P$. novaurelia (Fig. 2 ).

\section{Analysis of rDNA fragments and COI mtDNA gene}

Results are generally similar, independent of the sequenced gene fragment.

Analysis of fragments of COI mtDNA (660bp), 3'SSU- ITS1 rDNA (210bp), and 5' LSU rDNA (350bp) fragments by NJ and MP methods revealed the existence of three main strain clusters (Figs 3-6), one cluster composed of strains from Scotland, Poland, USA and the second composed of strains from Spain, the Czech Republic, Turkey and again one strain from Poland, as well as a third cluster containing the strain from France. P. caudatum was used an an outgroup.

Ribosomal DNA analysis shows 24 haplotypes within $P$. novaurelia strains, 5 in the 3'SSU-ITS1 fragment and 19 in the 5 ' LSU fragment (Table $3 a)$. The genetic distance between all the studied strains ( $P$. novaurelia and $P$. caudatum) in the first ribosomal fragment is at the level of $6.2 \%$, and between $P$. novaurelia as $2.4 \%$. The 5 'LSU fragment reveals higher diversity with $11.1 \%$ divergence between $P$. novaurelia and $P$. caudatum strains, and 5.4\% divergence among $P$. novaurelia strains. Generally, 9 polymorphisms appear

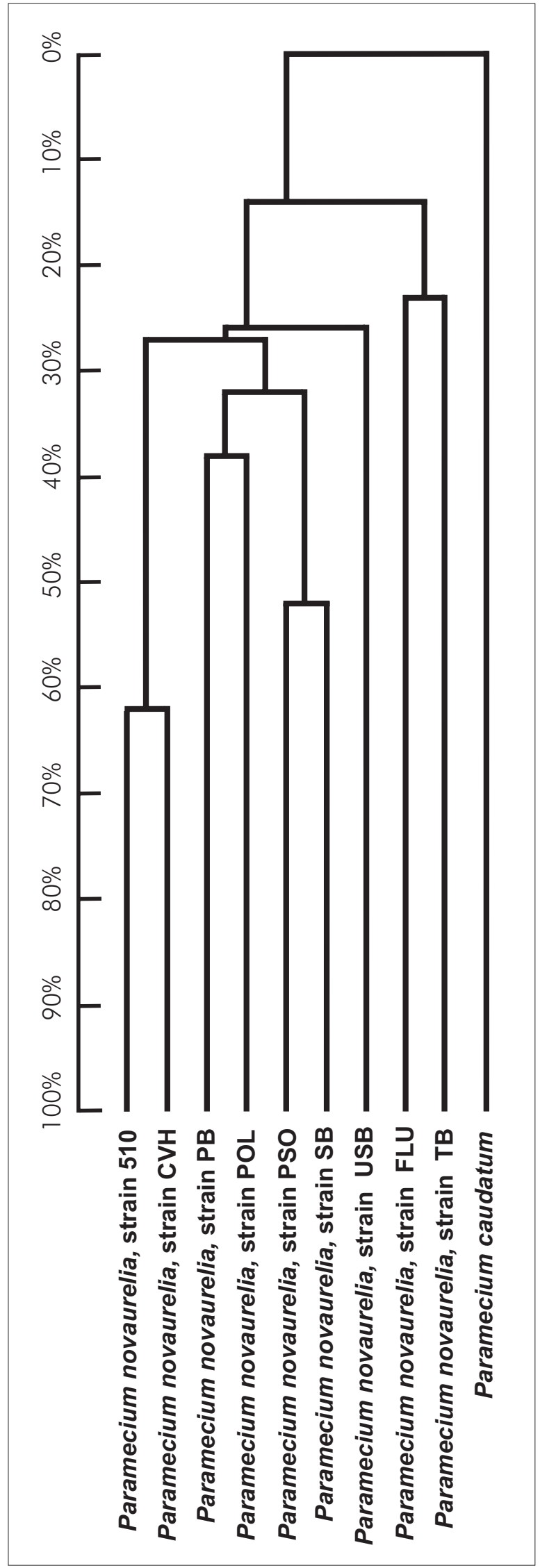

Fig. 2. Tree diagram of the cluster analysis of the RAPD fingerprint pattern similarity matrix of the studied $P$. novaurelia strains. Method used for analysis was UPGMA. Similarity index was calculated according to NEI and LI (1979). 

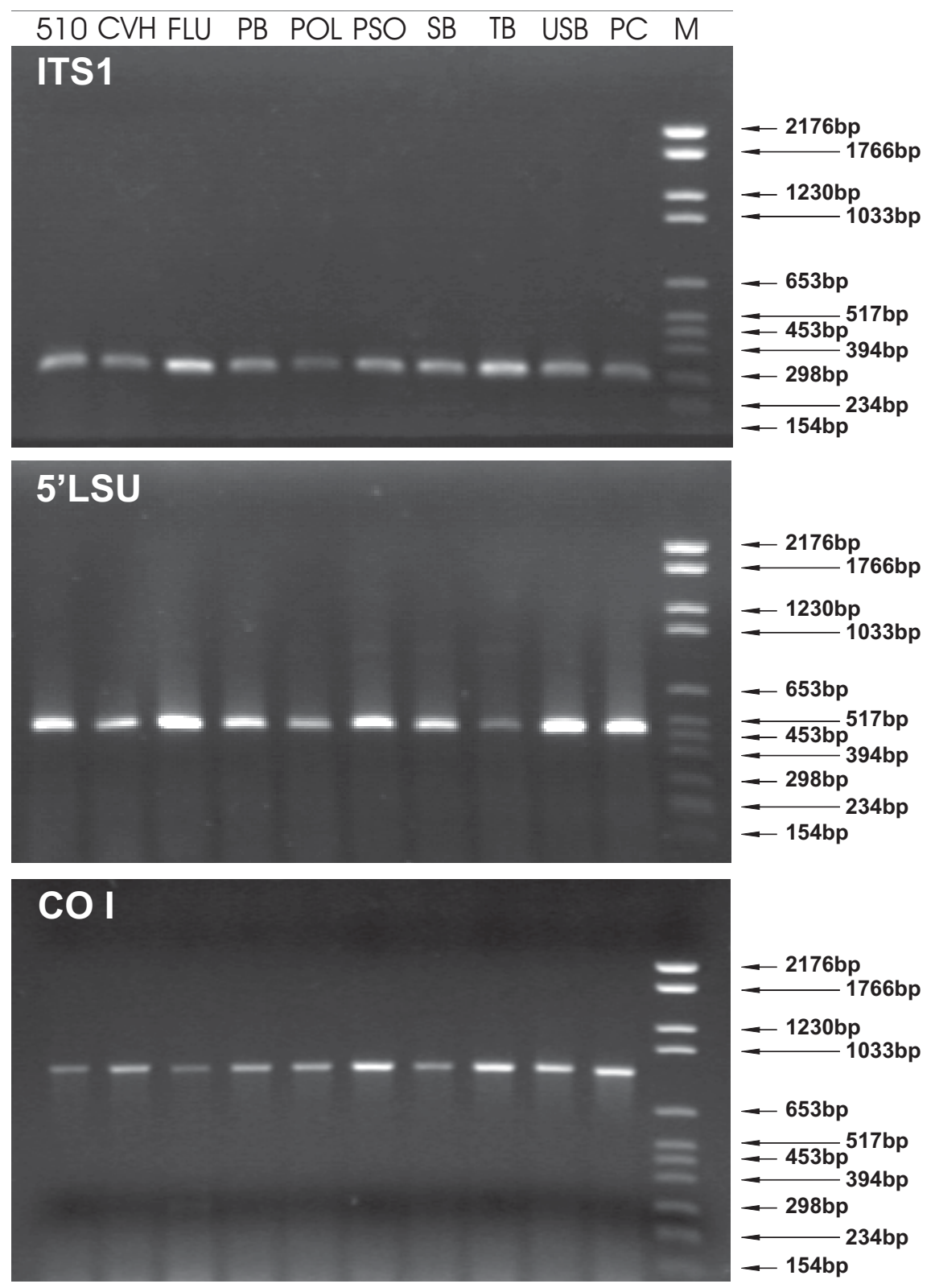

Fig. 3. Gel presenting the examined fragments: ITS1 - 3' end of the small subunit rRNA-internal transcribed spacer 1 fragment (310bp); 5'LSU - 5'end of the large subunit rRNA fragment (450bp); COI - fragment of COI gene (880bp). Designation of strains are the same as in Fig. 1a.

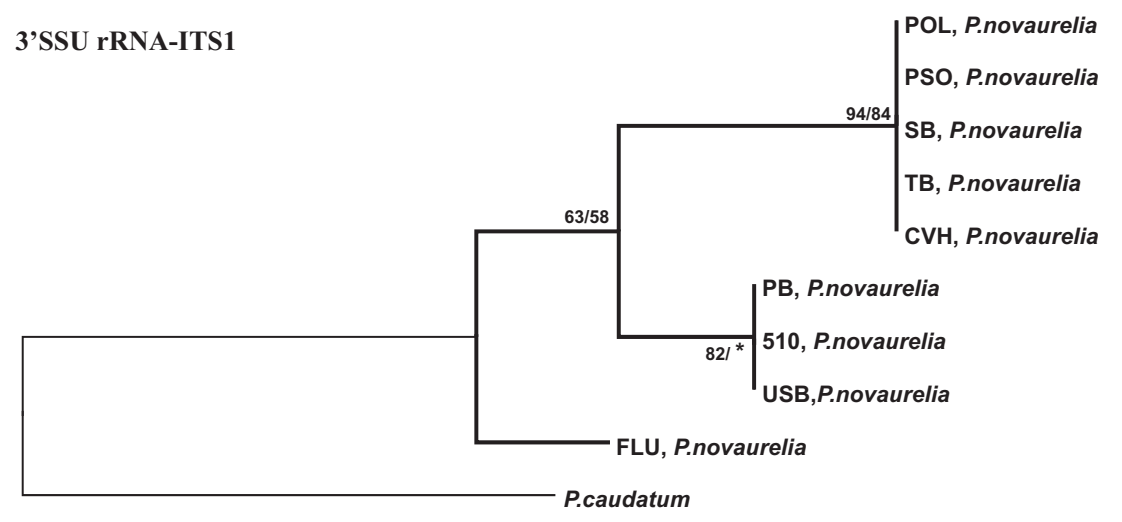

Fig. 4. Phylogenetic tree constructed for 9 strains of $P$. novaurelia and a single strain of $P$. caudatum as outgroup, based on a comparison of sequences from the 3' end of the small subunit rRNA-internal transcribed spacer 1 fragment using the NJ (neighbor joining) method with the application of the KIMURA two-parameter correction model and MP (maximum parsimony) analysis. Bootstrap values are presented as percentages (NJ/MP) for 100 comparisons. In the case of bootstrap values less than 50 , the asterisk appears. 


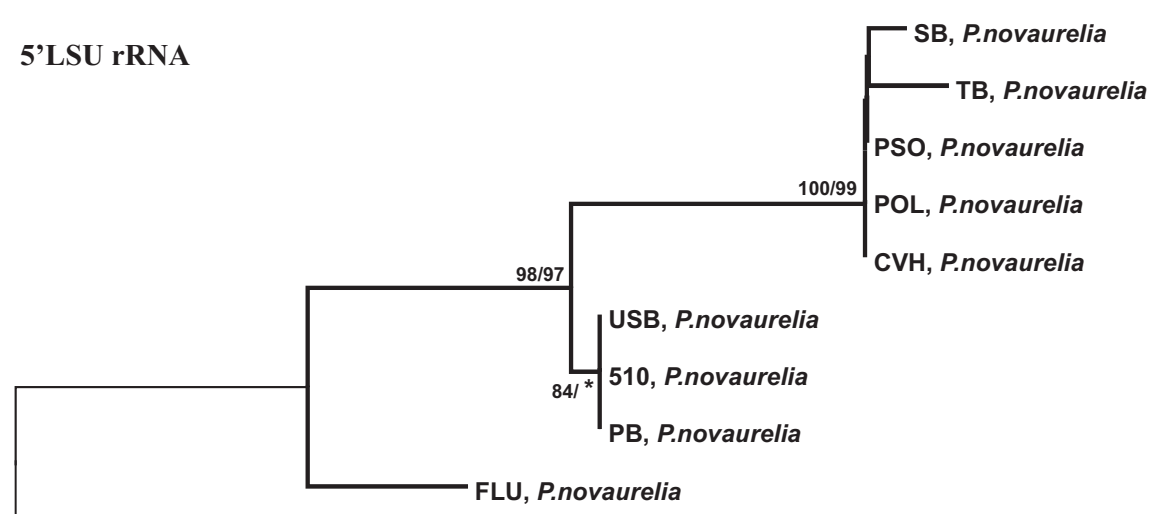

P. caudatum

Fig. 5. Phylogenetic tree constructed for 9 strains of $P$. novaurelia and a single strain of $P$. caudatum as outgroup, based on a comparison of sequences from the 5 'end of the large subunit rRNA fragment using the NJ (neighbor joining) method with the application of the KIMURA two-parameter correction model and MP (maximum parsimony) analysis. Bootstrap values are presented as percentages (NJ/MP) for 100 comparisons. In the case of bootstrap values less than 50, the asterisk appears.

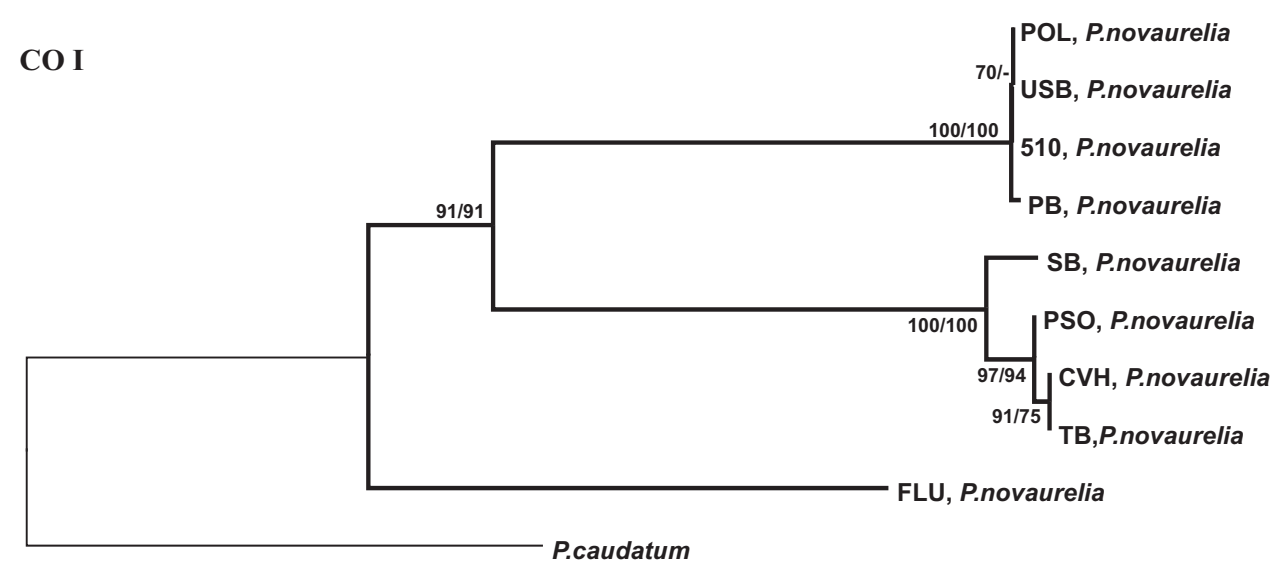

Fig. 6. Phylogenetic tree constructed for 9 strains of $P$. novaurelia and a single strain of $P$. caudatum as outgroup, based on a comparison of sequences from the fragment of CO I gene using the NJ (neighbor joining) method with the application of the KIMURA two-parameter correction model and MP (maximum parsimony) analysis. Bootstrap values are presented as percentages (NJ/MP) for 100 comparisons.

Table 3a

Polymorphisms in 3'SSU rRNA - ITS1 and 5'LSU rRNA fragments of $P$. novaurelia

\begin{tabular}{|c|c|c|c|c|c|c|c|c|c|c|c|c|c|c|c|c|c|c|c|c|c|c|c|c|}
\hline $\begin{array}{c}\text { Strain } \\
\text { designation }\end{array}$ & $\frac{N}{\#}$ & $\frac{6}{7}$ & $\frac{\infty}{\#}$ & $\frac{\infty}{\#}$ & $\begin{array}{l}\infty \\
\infty \\
\#\end{array}$ & $\frac{6}{m}$ & $\frac{2}{\#}$ & $\begin{array}{l}\text { 寺 } \\
\#\end{array}$ & $\frac{g}{ \pm}$ & $\begin{array}{l}n \\
\frac{n}{n} \\
\#\end{array}$ & $\begin{array}{l}\infty \\
n \\
\#\end{array}$ & 三 & $\frac{\mathrm{N}}{\#}$ & $\frac{2}{a}$ & $\begin{array}{l}\bar{\delta} \\
\text { స } \\
\#\end{array}$ & $\begin{array}{l}\text { ֻิ } \\
\text { ¿ } \\
\#\end{array}$ & $\begin{array}{l}n \\
\stackrel{n}{\#} \\
\#\end{array}$ & $\begin{array}{l} \pm \\
⿱ 亠 乂 \\
\#\end{array}$ & $\begin{array}{l}0 \\
N \\
\#\end{array}$ & $\begin{array}{l}\text { ป } \\
\#\end{array}$ & $\begin{array}{l}\mathfrak{n} \\
\stackrel{\sim}{v} \\
\#\end{array}$ & $\begin{array}{l}N \\
\text { N } \\
\#\end{array}$ & $\begin{array}{l}\stackrel{+}{\infty} \\
\text { N } \\
\#\end{array}$ & $\begin{array}{l}\bar{o} \\
m \\
\#\end{array}$ \\
\hline 510 & $T$ & C & $\mathbf{T}$ & $\mathbf{T}$ & $\mathbf{T}$ & $\mathbf{T}$ & $\mathbf{A}$ & $\mathbf{A}$ & $T$ & $\mathbf{A}$ & $\mathrm{C}$ & A & $\mathbf{G}$ & C & C & $\mathbf{G}$ & $\mathbf{G}$ & C & $\mathbf{G}$ & A & C & $\mathbf{G}$ & $\mathrm{C}$ & G \\
\hline $\mathrm{CVH}$ & C & $\mathbf{T}$ & . & . & A & . & . & . & . & . & . & $\mathbf{G}$ & $\mathbf{A}$ & $\mathbf{T}$ & $\mathbf{T}$ & . & C & . & . & . & $\mathbf{T}$ & $\mathbf{A}$ & . & $\mathbf{A}$ \\
\hline FLU & C & . & $\mathrm{C}$ & A & . & $\mathrm{C}$ & $\mathbf{G}$ & $\mathbf{G}$ & C & $\mathbf{T}$ & $\mathbf{T}$ & . & . & . & . & . & . & $\mathbf{T}$ & $\mathbf{A}$ & $\mathbf{G}$ & . & . & $\mathbf{T}$ & . \\
\hline PB & . & . & . & . & . & . & . & . & . & . & . & . & . & . & . & . & . & . & . & . & . & . & . & . \\
\hline POL & C & $\mathbf{T}$ & . & . & $\mathbf{A}$ & . & . & . & . & . & . & $\mathbf{G}$ & $\mathbf{A}$ & $\mathbf{T}$ & $\mathbf{T}$ & . & C & . & . & . & $\mathbf{T}$ & A & . & A \\
\hline PSO & C & $\mathbf{T}$ & . & . & $\mathbf{A}$ & . & . & . & . & . & . & $\mathbf{G}$ & $\mathbf{A}$ & $\mathbf{T}$ & $\mathbf{T}$ & & C & . & . & . & $\mathbf{T}$ & $\mathbf{A}$ & . & $\mathbf{A}$ \\
\hline SB & C & $\mathbf{T}$ & . & . & $\mathbf{A}$ & . & . & . & . & . & . & $\mathbf{G}$ & $\mathbf{A}$ & $\mathbf{T}$ & $\mathbf{T}$ & A & C & . & . & . & $\mathbf{T}$ & $\mathbf{A}$ & . & A \\
\hline TB & C & $\mathbf{T}$ & . & . & $\mathbf{A}$ & . & . & . & . & . & . & $\mathbf{G}$ & $\mathbf{A}$ & $\mathbf{T}$ & $\mathbf{T}$ & & C & . & . & . & $\mathbf{T}$ & $\mathbf{A}$ & . & A \\
\hline USB &. & . & . & . & . & . & . & . & . & . & . & . & . & . & . & . & . & . & . & . & . & . & . & . \\
\hline
\end{tabular}


Table 3b

Polymorphisms in COI mtDNA fragment of $P$. novaurelia

\begin{tabular}{|c|c|c|c|c|c|c|c|c|c|c|c|c|c|c|c|c|c|c|c|c|c|c|c|c|c|c|c|c|c|c|c|c|c|c|c|c|}
\hline & & & & & & & & & & & & & & & & & & & & & & & & & & & & & & & & & & & & \\
\hline P. novaurelia & $\boldsymbol{A}$ & & G $A$ & $\mathrm{C}$ & $\mathrm{C} \mathbf{C}$ & A & & C & A $\mathbf{C}$ & & & $\mathrm{T}$ & A & C & & $\mathbf{T}$ & A $\mathbf{C}$ & & $\begin{array}{ll}\mathbf{T} & \mathbf{T} \\
\end{array}$ & 厂 A & $T$ & $\mathbf{T}$ & C & C & $\mathbf{T}$ & $\begin{array}{ll}\mathbf{T} & \mathbf{T} \\
\mathrm{C}\end{array}$ & $\mathbf{T} \mathbf{C}$ & G C & $\mathbf{A}$ & $\mathbf{G}$ & & \begin{tabular}{l|l}
$\mathrm{C}$ & $\mathbf{T}$ \\
\end{tabular} & $\mathbf{T}$ & $\mathbf{A}$ & & \\
\hline P. novaurelia, strain $\mathrm{CVH}$ & G & 1 & $\mathbf{T} \mathrm{C}$ & & $\mathbf{T}$ & & C & $\mathbf{A}$ & & $\mathbf{T} \mathbf{T}$ & $\mathbf{T} \mid A$ & A & . & . & G & C & . & & . A & $\mathrm{G}$ & C & C & $\mathbf{T}$ & G & C & C. . & & A 1 & G & $\mathbf{A}$ & $\begin{array}{ll}\mathbf{A} & 1 \\
\end{array}$ & $\mathbf{T}$ C &. &. & & C \\
\hline P. novaurelia, strain FLU & $\mathbf{T}$ & & $\begin{array}{l}\text { A } \\
\end{array}$ & $\Gamma \mathrm{T}$ & $\begin{array}{r}\mathbf{r} \\
\mathbf{T}\end{array}$ & $\mathbf{T}$ & C & $\mathbf{T}$ & & $\mathbf{T} \mathbf{T}$ & $\mathbf{T} A$ & 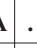 & C & $\mathbf{T}$ & $\mathrm{T}$ & $\cdot$ & G & & C A & $\mathrm{C}$ &. & C & $\mathbf{T}$ & $\mathbf{A}$ & $\mathbf{C}$ & .0 & C 7 & $\mathbf{T} \mathbf{T}$ & & . & . C & $\mathbf{G} \mathbf{A}$ & $\mathbf{A}$ & G & & . \\
\hline P. novaurelia, strain PB & . & . & . & . & . & . & & . & . & te & . & 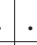 & . & . & . & . & . & Le &. & . & . & . & $\cdot$ &. &. & . & - &. & . &. &. &. &. &. & . & . \\
\hline P. novaurelic & . & . & . & . & . & - & & . & $\cdot$ & & - & 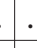 & . & . & . & . & . & & . & . & . & . &. & . & $\cdot$ & . & - & $\cdot$ & . &. & . & . &. & . & . & - \\
\hline P. novaurelia, strain PSO & . & & . C & & . $\mathbf{T}$ & . & C & $\mathbf{A}$ & & $\mathbf{T} \mathbf{T}$ & $\mathbf{T}$ : $A$ & $A$ & . & . & G & C & . & & . & $\mathrm{C}$ & C & C & $\mathbf{T}$ & G & $\mathbf{c}$ & $\mathbf{C}$. & & $\mathbf{A} \mathbf{T}$ & G & $\mathbf{A}$ & $\begin{array}{ll}\mathbf{A} & \mathrm{T} \\
\end{array}$ & $\mathbf{T}$ C &. &. & & $\mathbf{C}$ \\
\hline P. novaurelia, & . & & . C & & $T$ & - & C & $\mathbf{A}$ & -7 & T 7 & $\mathbf{T}$ A & $\mathrm{A}$ & . & . & G & C & . & & . A & $\mathrm{c}$ & C & C & $\mathbf{T}$ & G & C & $\mathbf{C}$ & & A 1 & G & $\mathbf{A}$ & \begin{tabular}{l|l}
$\mathbf{A}$ & $\mathbf{1}$ \\
\end{tabular} & $\begin{array}{ll}\mathbf{T} & \mathbf{C} \\
\end{array}$ & & $\mathbf{G}$ & & C \\
\hline P. novaurelia, strain ТВ & - & & $\mathrm{T} C$ & & $\mathbf{T}$ & - & C & A & & T 7 & $\mathbf{T}$ TA & $\mathrm{A}$ & . & . & $\mathbf{G}$ & C & . & & . $A$ & $\mathrm{C}$ & C & C & $\mathbf{T}$ & G & $\mathbf{c}$ & $\mathbf{C}$ & A & $\mathbf{A}$ & G & $\mathbf{A}$ & $\begin{array}{ll}\mathbf{A} & 1 \\
\end{array}$ & $\mathbf{T}$ C &. & & $\mathbf{G}$ & C \\
\hline P. novaurelia, strain USB & . & & . & . & . & . & & . & - & & . & - & . & . & • & . & . & & . & . & , & . & , & . & . & $\cdot$ & D. & . & 1 & . & $\cdot$. & . &. & & . & $\cdot$ \\
\hline & & & & & & & & 年 & $\begin{array}{l}\overrightarrow{7} \\
\#\end{array}$ & & & & & & & $\begin{array}{l}0 \\
\text { d } \\
\text { \# }\end{array}$ & 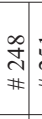 & & & & & & & 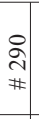 & $\begin{array}{l}\text { î } \\
\neq\end{array}$ & 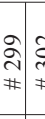 & & & & 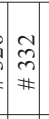 & હొ & & & & & $\begin{array}{l} \\
\text { m } \\
\text { \# }\end{array}$ \\
\hline P. novaurelia, strain 510 & $C$ & & G $C$ & $\mathrm{C} T$ & $\mathbf{T}$ & A & $\mathbf{T}$ & $\mathbf{T}$ & C & & $\begin{array}{lll}C & \end{array}$ & $\mathbf{I}$ & C & $\mathbf{T}$ & A & A & $\mathbf{G}$ & $\mathbf{A} A$ & $\begin{array}{lll}\mathbf{A} & \mathbf{1} \\
\end{array}$ & $\begin{array}{ll}\Gamma & \text { T } \\
\end{array}$ & $T$ & G & $\mathbf{T}$ & G & $\mathbf{T}$ & \begin{tabular}{l|l}
$\mathbf{C}$ & $\mathbf{T}$ \\
\end{tabular} & T & $G$ & & $\mathbf{T}$ & $\begin{array}{ll}\mathbf{T} & A \\
\end{array}$ & $\begin{array}{lll}A & C \\
\end{array}$ & C & $\mathbf{A}$ & & C \\
\hline P. novaurelia, strain $\mathrm{CVH}$ & . & $\mathbf{C}$. &. & & . & G & $\mathbf{A}$ & C & $\mathbf{T}$ & & T & $\mathrm{C}$ & T & C & - & . &. & C C & $\mathbf{G}$. & C & C & . & C &. &. & T C & C $A$ & $4 \mathrm{~T}$ & G & $\mathbf{C}$ & C C & $\begin{array}{ll}\mathbf{G} & \mathbf{G} \\
\end{array}$ &. & & . & - \\
\hline P. novaurelic & $\mathbf{T}$ & & $\begin{array}{lll}\mathbf{A} & \mathbf{T} \\
\end{array}$ & $\Gamma$ & $\mathbf{C} \mathbf{G}$ & & C & . & $\mathbf{T}$ & T & G & $\mathbf{C} A$ & . & . & G & $\mathbf{G}$ & $\mathbf{A}$ & G & G C & $\mathrm{C}$ & & $\mathbf{A}$ & & $\mathbf{A}$ & $\mathbf{C}$ & $\mathbf{T}$ & & $\mathbf{A}$ & & $\mathbf{A}$ & C C & $\begin{array}{lll} & \text { A } \\
\end{array}$ & & $\mathbf{T}$ & $\mathbf{T}$ & $\mathbf{T}$ \\
\hline P. novaurel & - & & . & . & . & . & & . & . & & . &. & . & . & . & $\cdot$ & $\cdot$ & &. & . & . & . &. &. & $\cdot$ &.$\cdot$ & . & .. & . &. &. & .. &. & . &. & . \\
\hline P. novaurelia, strain POL & . & & $\cdot$ & . & . & . & & $\cdot$ & . & & . &. & . & . & . & . &. & &. & . & . & . &. & . &. &. & &. & &. &. & .. &. & . & . & - \\
\hline P. novaurelia, strain PSO & - & C &. & . & . & G & $\mathbf{A}$ & C & $\mathrm{T}$ & & T & $\mathrm{C}$ & $\mathbf{T}$ & C & . & . &. & C C & \begin{tabular}{l|l}
$\mathbf{G}$. \\
\end{tabular} & C & C & . & C & . & . & T C & $\begin{array}{l}\mathbf{C} \\
\end{array}$ & $\begin{array}{ll}\mathbf{A} & \mathbf{T} \\
\end{array}$ & G & C & C C & $\begin{array}{lll}\mathbf{G} & \mathbf{G} \\
\end{array}$ &. & . & . & . \\
\hline P. novaurelia, strain $\mathrm{SB}$ & . & C & . & . & . & $\mathbf{G}$ & $\mathbf{A}$ & C & $\mathbf{T}$ & & T C & $C$ & $\mathrm{~T}$ & C & . & . & $\cdot$ & T & $\mathbf{G}$. & . & C & . & & $\cdot$ & . & T C & $\begin{array}{l}\mathbf{C} \\
\mathbf{C}\end{array}$ & $\begin{array}{ll}\mathbf{A} & \mathbf{T} \\
\end{array}$ & G & $\mathbf{C}$ & C C & $\begin{array}{ll}\mathbf{G} & \mathbf{G} \\
\end{array}$ & $\mathbf{T}$ & G & & . \\
\hline P. novaurelia, strain TB & . & C & . & . & . & $\mathbf{G}$ & $\mathbf{A}$ & C & $\mathbf{T}$ & 1 & T) & $\mathrm{C}$ & T & C & . & . &. & C & $\mathbf{G}$. & . & C & & C & . &. & T & C $A$ & $\begin{array}{ll}\mathbf{A} & \mathrm{T} \\
\end{array}$ & G & $\mathbf{C}$ & C C & $\begin{array}{l}\mathbf{G} \\
\mathbf{G}\end{array}$ & $\cdot$ & & . & . \\
\hline P. novaurelia, strain USB & & & - & . & . & . & & . & - & & - & $\cdot$ & . & . & . & . & $\cdot$ & & - & . & . & . & $\cdot$ & . & . & $\cdot$ & & . & . & . & $\cdot$. & . $\cdot$. & $\cdot$ & & . & . \\
\hline & & & & & & & & {$\left[\begin{array}{c}\tilde{D} \\
0 \\
\hdashline \\
\#\end{array}\right.$} & $\begin{array}{c}\infty \\
\infty \\
m \\
\neq\end{array}$ & & & & & & & $\begin{array}{l}\bullet \\
7 \\
\#\end{array}$ & $\begin{array}{c}0 \\
\vec{y} \\
\#\end{array}$ & $\begin{array}{c}\vec{y} \\
\nexists \neq \\
\#\end{array}$ & $\begin{array}{c}\tilde{y} \\
\neq\end{array}$ & & & & & $\begin{array}{l}n \\
\vdots \\
y \\
\# \\
\vdots\end{array}$ & $\begin{array}{c}\infty \\
0 \\
y \\
\neq \\
\neq\end{array}$ & $\begin{array}{l}\bar{b} \\
+ \\
\neq\end{array}$ & & & & 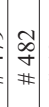 & 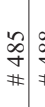 & & & & & $\begin{array}{c}\frac{N}{n} \\
\# \#\end{array}$ \\
\hline 10 & $C$ & & $\begin{array}{ll}C \\
C\end{array}$ & $\Gamma$ A & A C & A & C & A & C & & $\begin{array}{ll}C \\
C\end{array}$ & $\Gamma \mathrm{C}$ & c & $\mathbf{T}$ & $\mathbf{G}$ & $\mathbf{G}$ & $\mathbf{T}$ & $\mathbf{C}$ & $\begin{array}{ll}\mathbf{A} \\
\end{array}$ & $\begin{array}{lll}\Gamma & \mathbf{T} \\
\end{array}$ & $T$ & $\mathbf{A}$ & A & $\mathbf{A}$ & $\mathbf{A}$ & \begin{tabular}{l|l}
$\mathbf{T}$ & $\mathbf{T}$ \\
\end{tabular} & T & $\mathbf{G}$ & $T$ & $\mathbf{G}$ & $\begin{array}{lll}\mathbf{T} & \mathbf{T} \\
\end{array}$ & $\begin{array}{lll}\mathbf{T} & \mathbf{T} \\
\end{array}$ & $\mathbf{A}$ & & & $\mathbf{A}$ \\
\hline P. novaurelia, strain $\mathrm{CVH}$ & & $\mathbf{T}$ & $\mathrm{T}$ & $\mathrm{C}$ & $\begin{array}{ll}\mathbf{S} & \mathbf{T} \\
\end{array}$ & . & $\mathbf{T}$ & . & $\mathbf{T}$ & & T & $C$ & $\mathbf{T}$ & . & . & $\cdot$ & $\mathbf{C}$ & & 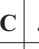 & . & G & C & G & G & $\mathbf{C}$ & C C & C C & \begin{tabular}{l|l}
$\mathbf{C}$ & $\mathbf{A}$ \\
\end{tabular} & C & . &. & .. & G & $\mathbf{T}$ & & T \\
\hline P. novaurelia, strain FLU & $\mathbf{T}$ & $T$ &. & $\mathrm{C}$ & . & C & & $\mathbf{T}$ & $\mathbf{T}$ & $\mathbf{T}^{\prime}$ & $T$ & . $\mathbf{T}$ & $T$ & C & $\mathrm{T}$ & A & $\mathbf{C}$ & $\mathbf{T}$ & $\mathbf{G} A$ & $\mathrm{~A}$ & C & . & $\cdot$ & G & $\mathbf{C}$ & . $A$ & $\mathbf{A}$. & - $\mathbf{A}$ & C & $\mathbf{A}$ & $\mathbf{C}$ & $\begin{array}{lll}\mathbf{A} & \mathbf{A} \\
\end{array}$ & & $\mathbf{T}$ & $\mathbf{A}$ & . \\
\hline P. novaurelia, strain PB & . & & . & . & . & . & & . & $\cdot$ & - & . & . & . & . & . & . & $\cdot$ & & . & . & . & . &. & $\cdot$ & $\cdot$ &. & . & $\begin{array}{l}\cdot . \\
\end{array}$ & . & $\cdot$ &. & . $\cdot$. &. & $\cdot$ & $\cdot$ & . \\
\hline P. novaurelia, strain POL & - & & $\cdot$ & . & . & . & & . & $\cdot$ & & . &. & . & . & . & . & . & &. & . & . & . &. & . &. &. & . & .. & . & . & .. & .. &. & . & $\cdot$ & . \\
\hline P. novaurelia, strain PSO & . & $\mathbf{T}$ & $\mathbf{T}$ & $\mathrm{C}$ & $\begin{array}{ll}G & T \\
\end{array}$ & . & $\mathbf{T}$ & $\cdot$ & $\mathrm{T}$ & . & T & $\mathrm{C}$ & T & . & . & . & C & & C & . & G & C & G & G & $\mathbf{C}$ & $\mathrm{C}$ & $\mathrm{C}$ & $\begin{array}{lll}C & A\end{array}$ & C & . & $\mathbf{C}$ & . & G & $\mathbf{T}$ & & $T$ \\
\hline P. novaurelia, strain SB & . & $\mathbf{T}$ & $\mathbf{T}$ & G & $\begin{array}{ll}\mathbf{S} & \mathrm{T} \\
\end{array}$ & . & $\mathbf{T}$ & . & $\mathbf{T}$ & 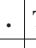 & T & $\mathrm{C}$ & $T$ & . & . & $\cdot$ & $\mathbf{C}$ & & $\mathbf{C}$. & . & $\mathbf{A}$ & C & G & G & $\mathbf{C}$ & C & C & \begin{tabular}{l|l}
$\mathbf{C}$ & $\mathbf{A}$
\end{tabular} & C & . & \begin{tabular}{l|l}
$\mathbf{C}$ & . \\
\end{tabular} & . & $\mathbf{G}$ & $\mathbf{T}$ & & $\mathbf{T}$ \\
\hline P. novaurelia, strain TB & . & $\mathbf{T}$ & $\mathbf{T}$ & $\mathrm{C}$ & $\begin{array}{ll}\mathbf{S} & \mathrm{T} \\
\end{array}$ & . & $\mathbf{T}$ & & $\mathbf{T}$ & & T & $\mathrm{C}$ & T & . & . & . & $\mathbf{C}$ & & $\mathbf{C}$. & . & G & C & G & G & $\mathbf{C}$ & C & C & $\begin{array}{lll}C & A\end{array}$ & C & . & $\begin{array}{ll}\mathbf{C} & \text {. } \\
\end{array}$ & . & G & $\mathbf{T}$ & & $T$ \\
\hline P. novaurelia & & . & $\cdot$ & • & . & . & & $\cdot$ &. & & . & .. & . & . &. & . & $\cdot$ & &. & . & . & . & $\cdot$ & $\cdot$ & . & $\cdot$. & & $\cdot \cdot$ & & . &. & $\begin{array}{lll}. & \text {. } \\
\end{array}$ &. & & & . \\
\hline & & & & & & & & 点 & in & & $\begin{array}{c}0 \\
\text { sh } \\
\text { wn }\end{array}$ & $\stackrel{n}{*}$ & & ñ & & ¿n & $\begin{array}{l}\bar{\infty} \\
i n\end{array}$ & & & 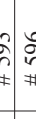 & & 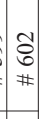 & $\mid \begin{array}{l}\infty \\
0 \\
0 \\
\neq\end{array}$ & $\begin{array}{l}\vec{z} \\
\neq \\
\#\end{array}$ & $\begin{array}{l} \pm \\
b \\
\neq\end{array}$ & $\begin{array}{l}6 \\
\neq\end{array}$ & & ชิ & & 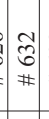 & $\begin{array}{c}\infty \\
0 \\
\vdots \\
\#\end{array}$ & $\begin{array}{c}\text { C్ } \\
* \neq\end{array}$ & 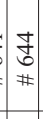 & & & \\
\hline P. novaurelia, strain 510 & $C$ & & A 1 & $\Gamma$ & $\Gamma$ & $\mathbf{T}$ & $\mathbf{T}$ & $\mathbf{T}$ & $\mathbf{G}$ & $\mathbf{T}$ & $\mathrm{C} C$ & $\mathrm{C}$ & A & G & $\mathbf{T}$ & $\mathbf{T}$ & $\mathbf{T}$ & $\mathbf{T}$ & $\mathrm{A}$ & $\mathrm{C}$ & $G$ & $\mathbf{A}$ & C & $\mathbf{T}$ & $\mathbf{C}$ & C C & C & $\mathrm{C}$ & $\mathbf{A}$ & $\mathbf{G}$ & $\begin{array}{ll}\mathbf{C} & \mathbf{T}\end{array}$ & $\begin{array}{lll} & G \\
\end{array}$ & $\mathbf{T}$ & $\mathbf{T}$ & & C \\
\hline P. novaurelia, strain $\mathrm{CVH}$ & $\mathbf{A}$ & & T & C & C & C & $\mathbf{A}$ & C & . & . & $\begin{array}{lll} & \\
\end{array}$ & $\Gamma$ & . & . & C & C & C & $\mathrm{C}$. & $\begin{array}{l}\mathbf{T} \\
\mathbf{T}\end{array}$ & $\begin{array}{ll}\Gamma & T \\
\end{array}$ & $\mathbf{A}$ & C & $\mathbf{T}$ & C & $\mathbf{A}$ & 1 & $\mathbf{T}$ & • & C & $\mathbf{A}$ & G & C . & A & $\mathbf{A}$ & & $\mathbf{T}$ \\
\hline P. novaurelia, strain FLU & $\mathbf{A}$ & $\mathbf{T}$ & $\cdot$ & $\mathrm{G}$ & $\mathbf{s}$. & C & $\mathbf{A}$ & $\mathbf{A}$ &. & $\mathbf{C}$ & $\begin{array}{l}\mathbf{A} . \\
\end{array}$ & T & $\mathbf{T}$ & $\mathbf{T}$ & & . & . & $\cdot$ & . & T & C & C & $\mathbf{T}$ & . & $\mathbf{T}$ & $\begin{array}{lll}\mathbf{T} & \mathbf{T} \\
\end{array}$ & $T$ & $\mathbf{T} \mathbf{A}$ & $\mathbf{T}$ & . & $\mathbf{T}$ & \begin{tabular}{l|l}
$\mathbf{C}$ & $\mathbf{A}$ \\
\end{tabular} & $\mathbf{G}$ & C & $\mathbf{C}$ & . \\
\hline P. novaurelia, strain $\mathrm{PB}$ & $\cdot$ & & $\cdot$ & • & . & . & & . & $\mathbf{A}$ & 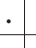 & . & . & . & . & . & . & . & $\cdot$ & . & . & . & . & $\cdot$ & . & . &. & - & . & . & . &. & . $\cdot$. & . &. & . & . \\
\hline P. novaurelia, strain POL & . & & $\cdot$ & . & - & - & & $\cdot$ & - & 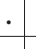 & . & . & . & . & . & . & . & & - & . & . & . &. & . & . & . & - & . & . & . &. . & .. &. & . & . & - \\
\hline P. novaurelia, strain PSO & $\mathbf{A}$ & & T C & C & . & C & & C & . & & T 1 & I A & . & . & C & C & C & & $\mathbf{T} 7$ & \begin{tabular}{r|r} 
& $T$ \\
\end{tabular} & A & C & $\mathbf{T}$ & C & $\mathbf{A}$ & . 1 & $\mathbf{T}$ & $\cdot$ & C & $\mathbf{A}$ & G C & C. & $\mathbf{A}$ & $\mathbf{A}$ & & $\mathbf{T}$ \\
\hline P. novaurelia, strain $\mathrm{SB}$ & $\mathbf{A}$ & & $\mathbf{T}$ & c) & . $\mathrm{C}$ & . & A & C & . & & T 1 & I A & 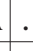 & . & C & C & C & C & G $\mathbf{T}$ & $\begin{array}{r}\Gamma \\
\end{array}$ & A & C & $\mathbf{T}$ & C & $\mathbf{A}$ & & T & • & C & A & G C & C. & $\mathbf{A}$ & $\mathbf{A}$ & $\mathbf{A}$ & . \\
\hline P. novaurelia, strain TB & $\mathbf{A}$ & & T C & c & C & C & & C & $\cdot$ & & T 1 & I A & 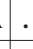 & . & C & C & C & C & T 7 & $\Gamma$ & A & C & $\mathbf{T}$ & C & $\mathbf{A}$ & & T & & C & $\mathbf{A}$ & G C & C . & $\mathbf{A}$ & $\mathbf{A}$ & & $\mathbf{T}$ \\
\hline P. novaurelia, strain USB & & & & & & . & & & $\cdot$ & & & . & & . & & & . & & & & & & & & & & & & & &. & & & & & \\
\hline
\end{tabular}


in both fragments, grouping the examined strains into two main clusters, and there are also 12 haplotypes characteristic only for the FLU strain.

COI mtDNA analysis. Much higher divergence was observed in the case of the COI mtDNA fragment, 215 haplotypes (for all studied strains) and 164 substitutions were found in the $P$. novaurelia strains comparison (Table $3 \mathrm{~b}$ ). The distance between $P$. novaurelia and $P$. caudatum strains is about $32.7 \%$, and $24.8 \%$ among $P$. novaurelia strains.

When $P$. novaurelia was compared with the other Paramecium spp. (P. caudatum, P. multimicronucleatum, $P$. schewiakoffi) some variable positions characteristic for all studied species of the genus were found. A geographical correlation between genotypes was not observed, and most haplotypes occurred at the third codon position.

\section{Discussion}

Polymorphism within $P$. novaurelia, appearing as differentiated RAPD fingerprints (genotypes), was first revealed by STOECK et al. (2000). The genotypes seemed not to be closely associated with the geographical origin of the studied strains, also a high percentage of surviving clones in $\mathrm{F}_{1}$ and $\mathrm{F}_{2}$ generations was observed in inter-strain hybrids. Later, RAPD fingerprints of the newly identified strains of $P$. novaurelia from Russia were compared with band patterns of other European strains of the species and intra-specific polymorphism was confirmed (PRZYBOŚ et al. 2006b). Several genotypes within $P$. novaurelia were also observed in the present study when strains originating from different continents (North America, Europe, and Asia) were compared, and two main groups of strains were revealed (Figs 1a,b, 2). The band pattern characteristic for $P$. novaurelia is different from the band pattern of Paramecium caudatum.

STOECK et al. $(1998,2000)$ suggested that such polymorphism may be associated with a degree of inbreeding which is characteristic (SONNEBORN 1957; LANDIS 1986) for the species of the complex and may cause intra-specific differentiation. This correlation was confirmed by studies concerning polymorphism within $P$. dodecaurelia (PRZYBOŚ et al. 2005) and by studies carried out on several strains of other species of the $P$. aurelia complex (PRRZYBOŚ et al. 2006 a). Species characterized by inbreeding (e.g. P. tetraurelia, P. dodecaurelia) showed higher intra-specific polymorphism in band pattern than did species characterized by weak inbreeding such as $P$. pentaurelia.

Polymorphism within $P$. novaurelia revealed by the RAPD method was in turn confirmed and analyzed by comparison of rDNA and COI mtDNA gene sequences. As a result, the existence of vari- ous strain clusters within species was revealed, however they are different from those obtained by RAPD analysis, only the distinctness of the FLU strain is similar. Lack of correlation (revealed by analysis of rDNA fragments and COI fragment of mtDNA) between different strain genotypes and their distribution (geographical origin) is in consensus with previous studies carried out by STOECK et al. (2000) with application of a RAPD marker. Perhaps this may be connected with the manner of expansion (spreading) of paramecia around the world, they can be transported for long distances by birds (SONNEBORN 1957; COLEMAN 2005).

Intra-specific polymorphism within $P$. novaurelia was at a level of 2.4\% in 3'SSU-ITS1 and 5.4\% in 5 ' LSU fragments of rDNA as well as $13.9 \%$ divergence in the case of COI mtDNA. Many highly variable positions and polymorphic sites characteristic for the strain groups (Table $3 b$ ) were found in COI mtDNA in all studied strains of $P$. novaurelia as well as in other species of the Paramecium genus. Three polymorphic sites were localized in single stranded nucleotide positions of ITS1. A much higher divergence was observed in the case of the COI mtDNA fragment than in the rDNA fragments. It is worth mentioning that the fragment of COI mtDNA investigated here has been recently used as a bar-code, i.e a standard fragment of DNA appearing in the majority of living organisms enabling the analysis of phylogenetic relationships on low and high taxonomic levels (HEBERT et al. 2003). However, our analyses showed the limited usefulness of this mtDNA fragment for investigations of broad relationships, it seems useful on rather low taxonomic levels as revealed by the high variability at intra-specific or intra-population levels. Analysis of the COI mtDNA fragment revealed intra-specific differentiation not only in Paramecium but also in the butterfly Astraptes fulgerator (HEBERT et al.2004), as the existence of ten cryptic species within the previous single species.

Information on intra-specific molecular diversity within $P$. aurelia species is rare. However, studies carried out within $P$. dodecaurelia showed that its intra-specific diversity was as high as that between different species of the $P$. aurelia complex based on analysis of sequences of rDNA fragments, i.e. six polymorphic sites were found in a fragment of rRNA at the 3' end of SSU and several sites at the 5' end of LSU (TARCZ et al. 2006). In $P$. novaurelia 24 polymorphisms appear in both rRNA fragments. Similarly, analysis of sequences of hsp70 (HORI et al. 2006) and the H4 histone (PRZYBOŚ et al. 2006c) genes also showed the isolated position of $P$. dodecaurelia within the tree constructed for species of the $P$. aurelia complex. Intra-specific differentiation within $P$. novaurelia is not as substantial as that existing within 
P. dodecaurelia. Intra-specific polymorphism was also studied in P. quadecaurelia (PRZYBOŚ et al. 2003b) by comparison of cytosol-type hsp 70 gene sequences but the base sequence of both strains were $99.2 \%$ identical.

Recently, the mitochondrial cytochrome c oxidase I (COI) gene was used for intraspecific investigations in $P$. caudatum and $P$. multimicronucleatum and results were compared with obtained sequences of the ITS regions (BARTH et al. 2006). The mitochondrial sequences revealed higher variation in both species with intra-specific divergences up to $7 \%$ in P. caudatum and $9.5 \%$ in P. multimicronucleatum. $13.9 \%$ divergence in COI mtDNA was found in $P$. novaurelia strains in this study.

Several studies concerned comparisons of species within the $P$. aurelia complex.

NANNEY et al. (1998) compared sequence differences in a variable 23S rRNA domain among several species of the $P$. aurelia complex (without P.novaurelia) and found that pairs of species are separated by four or more changes. Intra-specific differentiation was not studied but the authors wrote "since multiple clones of the same species were not examined, D2 polymorphism within aurelia species remains a possibility". Here, polymorphism within $P$. novaurelia was revealed. To evaluate the level of polymorphism in $P$. novaurelia, the variabilty should be compared with results of other studies carried out on the P. aurelia complex with the application of SSU rRNA gene sequences, e.g. $P$. primaurelia and $P$. tetraurelia differed by five nucleotides from each other (STRÜDER-KYPKE et al. 2000a,b). Another species of the Paramecium genus, Paramecium schewiakoffi, had $99.3 \%$ similarity with the sequences of $P$. jennningsi and a $98.9 \%$ similarity with sequences of $P$. tetraurelia and P. primaurelia (FOKIN et al. 2004) based on $18 \mathrm{~S}$ rRNA sequences. In turn, COLEMAN (2005) investigated the rRNA ITS region in the $P$. aurelia complex and found strain variability as "variant nucleotide positions" only within P. primaurelia and $P$. tredecaurelia. P. tetraurelia and $P$. novaurelia and also $P$. octaurelia and $P$. dodecaurelia were identical in the entire ITS2 sequence. However, the author only studied no more than two strains of the particular species. Hsp 70 gene sequences were also used by HORI et al. (2006) for comparison of species of the $P$. aurelia complex and $P$. caudatum syngens. They found that non-synonymous substitutions were frequent in $P$. triaurelia, P. septaurelia, P. dodecaurelia $(11,10$, 5 respectively in these species), $P$. novaurelia, $P$. tredecaurelia, and $P$. quadecaurelia have also one non-synonymous substitution in the same position. However, the authors did not study intraspecific differentiation.

In the future, application of the ITS2 fragment of rRNA in investigations concerning the intra- specific differentiation within Paramecium species of the aurelia complex will bring new data.

\section{Acknowledgements}

The authors are grateful to Dr S. FoKIN, Biological Research Institute, St. Petersburg State University, Russia, at present at the Department of Etology, Ecology and Evolution, University of Pisa, Italy for strains from the USA: YR (Yellowstone National Park) and BR (Baton Rouge).

\section{References}

Aufderheide K. J., DagGetT P.-M., Nerad T. A. 1983. Paramecium sonneborni n. sp., a new member of the Paramecium aurelia species-complex. J. Protozool. 30: 128-131.

BARTH D., KRENEK S, FOKIN S. I., BERENDONK T. U. 2006. Intraspecific genetic variation in Paramecium revealed by mitochondrial cytochrome $\mathrm{C}$ oxidase I sequences. J. Eukaryot. Microbiol. 53: 20-25.

BeAle G. H., SCHNELler M. 1954. A ninth variety of Paramecium aurelia. J. Gen. Microbiol. 11: 57-58.

CHEN T. T. 1956. Varieties and mating types in Paramecium bursaria. II. Variety and mating types found in China. J. exp. Zool. 132: 255-268.

COLEMAN A.W. 2005. Paramecium aurelia revisited. J. Euk. Microbiol. 52: 68-77.

FELSENSTEIN J. 1985. Confidence limits on phylogenies: an approach using the bootstrap. Evolution 39: 783-791.

HALL T. A. 1999. BioEdit: a user-friendly biological sequence alignment editor and analysis program for Windows 95/98/NT. Nucl. Acids, Symp. Ser. 41: 95-98.

Hebert P. D. N., CYWINSKa A., BALl S. L., DEWAARD J. R. 2003. Biological identifications through DNA barcodes. Proc. R. Soc. Lond. B. 270: 313-322.

Hebert P. D. N., PEnTON E. H., Burns J. M., JANZEN D. H., HALLWACHS W. 2004. Ten species in one: DNA barcoding reveals cryptic species in the neotropical skipper butterfly Astraptes fulgerator. Proc. Natl Acad. Sci. USA 101: 14 812-14 817.

Hori M., TOMIKAWA I., PrzyBoś E., FuJishima M. 2006. Comparison of the evolutionary distances among syngens and sibling species of Paramecium. Mol. Phylogenet. Evol. 38: 697-704.

JEROME C. A., LYNN D. H. 1996. Indentifying and distinguishing sibling species in the Tetrahymena pyriformis complex (Ciliophora, Oligohymenophorea) using PCR/RFLP analysis of nuclear ribosomal data. J. Euk. Microbiol. 43: 492-497.

KIMURA M. 1980. A simple method for estimating evolutionary rates of base substitutions through comparative studies of nucleotide sequences. J. Mol. Evol. 16: 111-120.

Komala A., KościuszKo H., HuMiCzEwSKA M. 1960. Further investigations $\mathrm{n}$ the occurrence of different varieties on Paramecium aurelia in Poland. Folia biol. (Kraków) 8: 59-63.

KOMALA Z., PRZYBOŚ E. 1989. Further studies on the species of the Paramecium aurelia complex in the Middle Sudetes of Poland. Folia biol. (Kraków) 37: 25-44.

KUMAR S., TAMURA K., NEI M. 2004. MEGA3: Integrated software for molecular evolutionary genetic analysis and sequence alignment. Briefings in Bioinformatics 5: 150-163.

LANDIS W. G. 1986. The interplay among ecology, breeding systems, and genetics in the Paramecium aurelia and Paramecium bursaria complexes. (In: Progress in Protistology, J.O. Corliss, D. Patterson. eds. Biopress, Bristol): 287-307. 
NANNEy D. L., PARK C., Preparata R., SimOn E. M. 1998. Comparison of sequence differences in a variable 23S rRNA domain among sets of cryptic species of ciliated protozoa. $\mathrm{J}$. Euk. Microbiol. 45: 91-100.

NEI M., LI W.-H. 1979. Mathematical model for studying genetic variation in terms of restriction endonucleases. Proc. Natl. Acad. Sci. 76: 5260-5273.

PRZYBOŚ E. 1975. Genetic studies of Paramecium jenningsi strains (Diller, Earl, 1958). Folia biol. (Kraków) 23: 425-471.

PRZYBOŚ E. 1978. Cytological and karyological studies of Paramecium jenningsi. Folia biol. (Kraków) 26: 25-29.

PRZYBOŚ E. 1991. Studies on the Paramecium aurelia species complex in Spain (Ciliophora) Arch. Protistenkd 140: 151-156.

PRZYBOŚ E. 1998. The first habitat of Paramecium novaurelia of the $P$. aurelia spp. complex in Asia (Turkey) Folia biol. (Kraków) 46: 91-95.

PRZYBOŚ E., FOKIN S. 2000. Data on the occurrence of species of the Paramecium aurelia complex world-wide. Protistology 1: 179-184.

PrZyboś E., FoKin S., STOECK T., SCHMIDT H. J. 1999. Occurrence and ecology of Paramecium jenningsi strains. Folia biol. (Kraków) 47: 53-59.

PRZYBoŚ E., HORI M., FoKIN S. I. 2003b. Strains of Paramecium quadecaurelia from Namibia, Africa. Genetic and molecular studies. Acta Protozool. 42: 357-360.

PRZYBoŚ E., Komala Z. 1992. The occurrence of the Paramecium aurelia species complex in the Eastern Sudetes. Folia biol. (Kraków) 40: 57-60.

PRZYBoŚ E., Komala Z. 1996. Notes on the Paramecium aurelia species complex in the region of the Orawa Valley. Folia biol. (Kraków) 44: 47-50.

PRZYBoŚ E., MACIEJEWSKA A., SKOTARCZAK B. 2006c. Relationships of species of the Paramecium aurelia complex (Protozoa, Ph. Ciliophora, Cl. Oligohymenophorea) based on sequencing of histone $\mathrm{H} 4$ gene fragment. Folia biol. (Krakow) 54: 37-42.

PrZyboŚ E., Prajer M., GRECZEK-STACHURA M., FOKIN S. I., RAUTIAN M., POTEKHIN A. 2005. New European stands of Paramecium pentaurelia, Paramecium septaurelia, and Paramecium dodecaurelia, genetic and molevular studies. Folia biol. (Kraków) 53: 123-128.

PrZyboś E., PRAJER M., GRECZEK-STACHURA M., SKOTARCZAK B., MACIEJEWSKA A., TARCZ S. 2006a. Genetic analysis of the Paramecium aurelia complex by classical and molecular methods. Systematics and Biodiversity (in press).

PRZYBoŚ E., RAUTIAN M., POTEKHIN A. 2004. First record of Paramecium septaurelia and the discovery of new European habitats of $P$. pentaurelia and $P$. sexaurelia in Russia (Astrahan and Volgograd regions). Folia biol. (Kraków) 52: 87-90.

PrzyboŚ E., Rautian M., POTEKHIN A. 2005. Species of the Paramecium aurelia complex in Russia, Lower Volga Basin. Protistology 4: 129-134.

Przyboś E., Skotarczak B., Wodecka B. 2003a. Phylogenetic relationships of Paramecium jenningsi strains (clas- sical analysis and RAPD studies). Folia biol. (Kraków) 51: 185-195.

PrZyboś E., TARCZ S., Rautian M., Potekhin A. 2006b. Species of the Paramecium aurelia complex in Russia (Western region of the European part) with molecular characteristics of $P$. novaurelia. Folia biol. (Kraków) 54:43-47.

SAITOU N., NEI M. 1987. The neighbor-joining method: a new method for reconstructing phylogenetic trees. Mol. Biol. Evol. 4: 406-425.

Skotarczak B., Przyboś E., Wodecka B., MacieJEWSKA A. 2004a. Sibling species within Paramecium jenningsi revealed by RAPD. Acta Protozool. 43: 29-35.

SKOTARCZAK B., PRZYBoŚ E., WODECKA B., MACIEJEWSKA A. 2004b. Random amplified polymorphic DNA fingerprint as a marker for Paramecium jenningsi strains. Folia biol. (Kraków) 52: 117-124.

SONNEBORN T. M. 1957. Breeding systems, reproductive methods and species problem in Protozoa. (In: The Species Problem, E. Mayr . ed. AAAS, Washington D.C.): 155-324.

SONNEBORN T. M. 1970. Methods in Paramecium research. (In: Methods in Cell Physiology, Prescott D.M. ed., vol. 4. Academic Press, New York, London): 241-339.

SONNEBORN T. M. 1975. The Paramecium aurelia complex of fourteen sibling species. Trans. Amer. Micros. Soc. 94: $155-178$.

Stoeck T., Przyboś E., Kusch J., SchmidT H. J. 2000. Intra-species differentiation and level of inbreeding of different sibling species of the Paramecium aurelia complex. Acta Protozool. 39: 15-22.

StOeCK T., PRZYBoŚ E., SCHMIDT H. J. 1998. A comparison of genetics with inter-strain crosses and RAPD-fingerprints reveals different population structures within the Paramecium aurelia complex. Europ. J. Protistol. 43: 348-355.

STOECK T., SCHMIDT H. J. 1998. Fast and accurate identification of European species of the Paramecium aurelia complex by RAPD-fingerprints. Microb. Ecology 35: 311-317.

STRÜDER-KYPKE M. C., WrigTH A.-D. G., FoKIN S. I., LYNN D. H. 2000a. Phylogenetic relationships of the genus Paramecium inferred from small subunit rRNA gene sequences. Mol. Phylogenet. Evol. 14: 122-130.

STRÜDER-KYPKE M. C., WRIGTH A.-D. G., FOKIN S. I., LYNN D. H. 2000b. Phylogenetic relationships of the subclass Peniculia (Oligohymenophorea, Ciliophora) inferred from small subunit rRNÅ gene sequences. J. Euk. Microbiol. 47: 419-429.

TARCZ S., PrZyboś E., Prajer M.,GreCZEK-STAChuRa M. 2006. Intraspecific variation of diagnostic rDNA genes in Paramecium dodecaurelia, P. tredecaurelia and P. quadecaurelia (Ciliophora, Oligohymenophorea). Acta Protozool. 45: $255-263$.

ThOMPSON J. D., Higgins D. G., GiBSON T. J. 1994. CLUSTAL W: improving the sensitivity of progressive multiple sequence alignment through sequence weighting, position-specific penalties and weight matrix choice. Nucleic Acids Res. 22: 4673-80.

VIVIER E. 1974. Morphology, taxonomy and general biology of the genus Paramecium. (In: Paramecium, A Current Survey. W.I.van Wagtendonk ed. Elsevier, Amsterdam): 1-89. 